\title{
An ambulance helicopter for emergency calls
}

\author{
N. SELLWOOD
}

Department of Accident and Emergency Medicine, Royal Cornwall Hospital, Infirmary Hill, Truro, Cornwall

\section{SUMMARY}

The use of a helicopter as a primary response vehicle for the Cornwall Ambulance Service is presented. A brief analysis of the activities of the First Air Ambulance is described and an appraisal of its effects on the overall performance of the Service is given. Emphasis is given to patient acceptability and also to the flexibility of the helicopter in terms of its response to different situations. In conclusion, The Air Ambulance, as part of an integrated ambulance service, is an effective provider of good pre-hospital care.

\section{INTRODUCTION}

The use of helicopters for the transport of patients is becoming increasingly common in the U.K. Most helicopter services only offer inter-hospital transport but since April 1987 the Cornwall Ambulance Service has been using a helicopter for the transport of patients in the pre-hospital phase.

The Cornwall Air Ambulance does not carry a doctor but is staffed by two ambulance personnel, at least one of whom has extended trained. The relatively low incidence of major trauma is largely the reason for not carrying a doctor and is in contrast with the London HEMS, and helicopter services in other countries where doctors are carried routinely (Baxt \& Moody, 1983; Baxt et al., 1985; Flora, 1985; Hossli, 1985; Herve et al., 1987; Moylan, 1988).

At its inception the Air Ambulance was available 5 days a week (including weekends), but as funding became more secure the service was increased to 7 days a week in July 1989 and with a longer working day. The number and frequency of missions is shown in Fig.1. The Air Ambulance is based in Truro and activated from there by Ambulance Control, delivering patients to any one of three District

Correspondence: N. Sellwood, Consultant, Department of Accident and Emergency Medicine, Royal Cornwall Hospital, Infirmary Hill, Truro TR1 2HZ, Cornwall. 


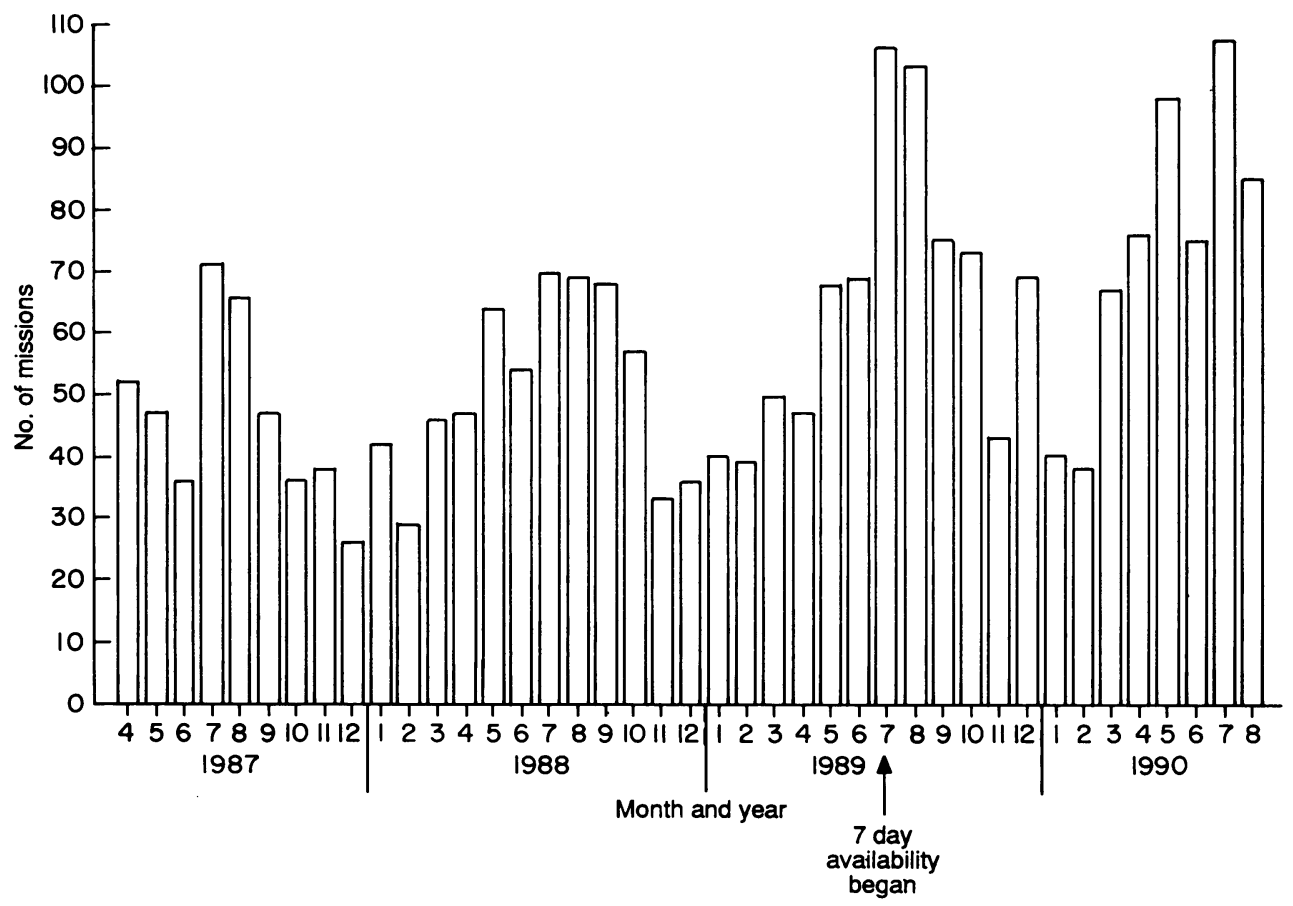

Fig. 1. Number of missions per month (April 1987-Aug 1990).

General Hospitals at Truro, Plymouth and Barnstaple. The map (Fig. 2) indicates approximate expected response times when flying from Truro and it can readily be seen that over virtually the whole of the County, a response can be achieved inside $20 \mathrm{~min}$. Helicopter landing pads are present at Derriford Hospital, Plymouth, at North Devon District Hospital, Barnstaple and at the Royal Cornwall Hospital (Treliske) in Truro. A large metallic surfaced, illuminated landing area is desirable but not necessary, and safe landings can be made on small grass areas or in car parks close to the A\&E entrance, when the patient's condition is critical (Fig. 3).

\section{Function}

The Air Ambulance, an MBB Bolkow 105B, has been especially adapted and is equipped to the specification laid down by the Cornwall Ambulance service, but based broadly on the $\mathrm{W}$. German experience. The interior is compact and well laid out giving easy access to the principal patient lying in the port bay. On the bulkhead above the patient, monitoring, suction, oxygen and defibrillation equipment is immediately to hand.

Further equipment is stowed inside rucksacks so that it can be carried away from the aircraft to the patient's side; these rucksacks are carried on the starboard bay stretcher routinely, and comprise splintage, infusion and airway control elements (Fig. 4). In the unusual event of two patients being carried (in only 32 of first 1165 missions), excess equipment can be either restowed elsewhere or left 


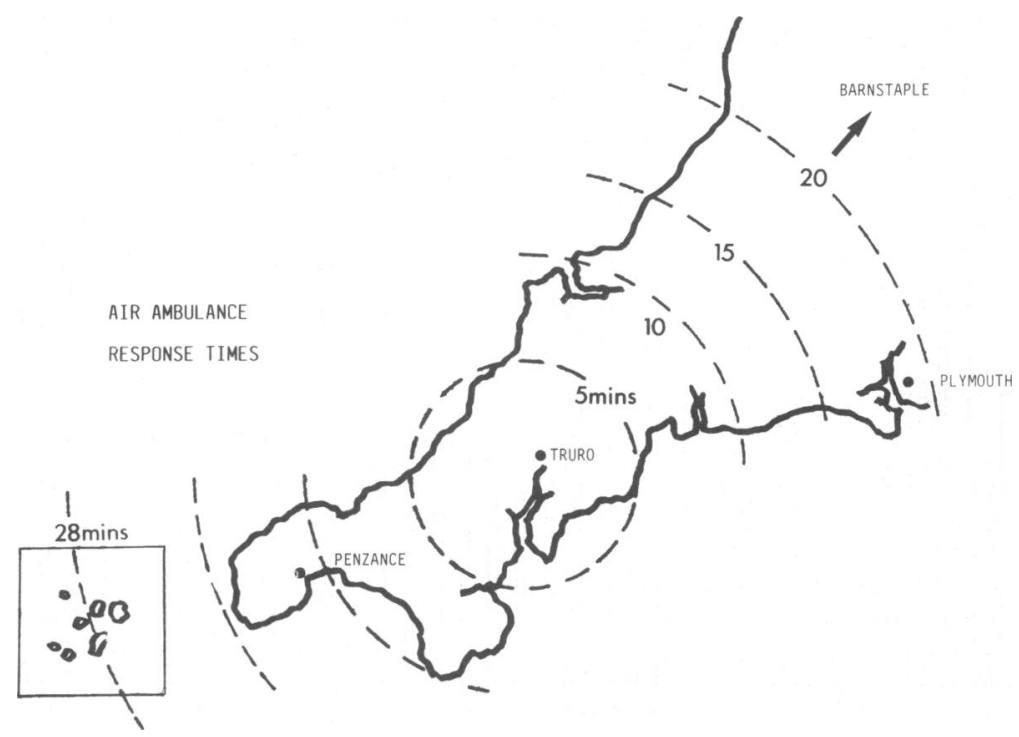

Fig. 2. Approximate air ambulance response times

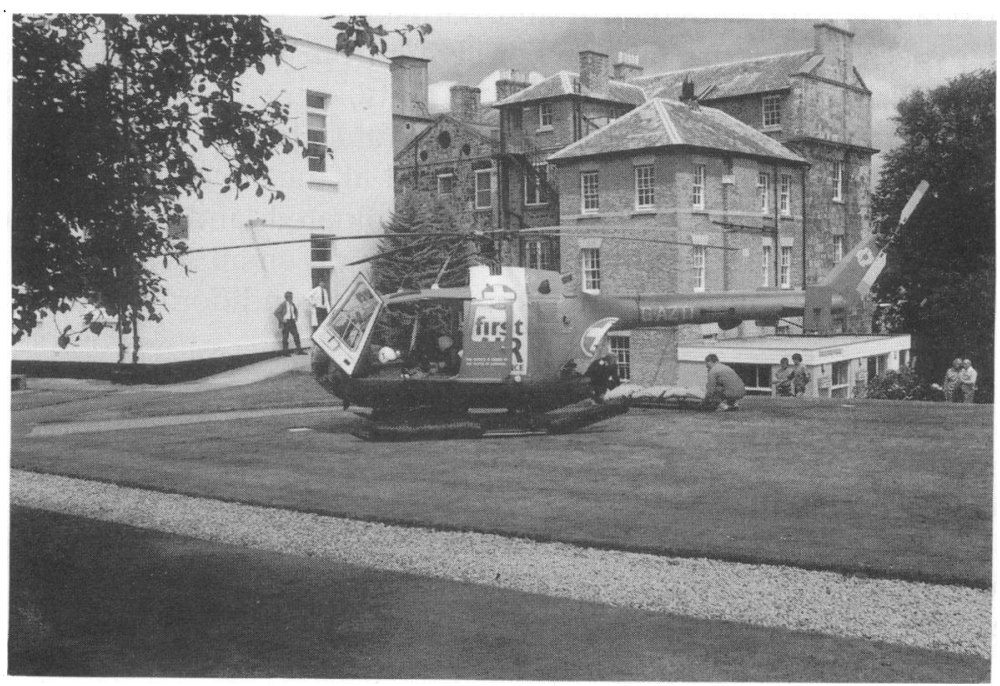

Fig. 3. Safe landings can be made close to the A\&E entrance when the patient's condition is critical. 


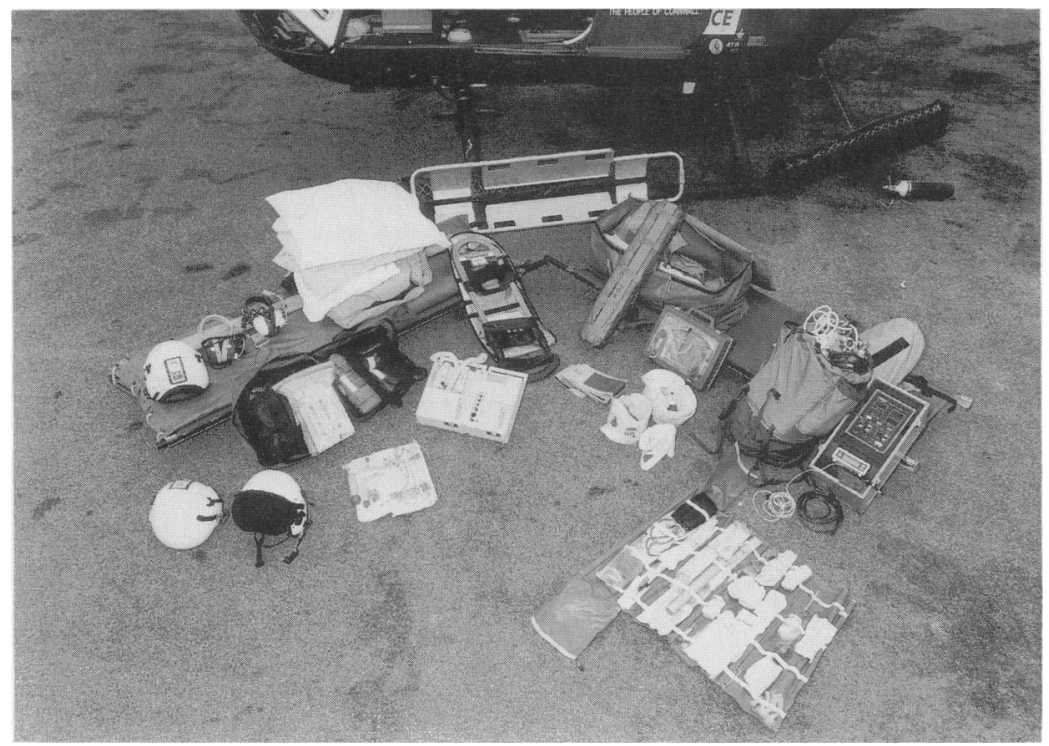

Fig. 4. Rucksacks comprising splintage, infusion and airway control elements are stored on the starboard bay stretcher so that they can be carried from the aircraft to the patient.

at the scene for later transport back to base by road. During the outward flight the extended trained ambulance man or 'paramedic' sits next to the pilot facing forwards and assists with pre-flight checks, navigation and radio communications. The other crew man sits facing sideways at all times. When a patient is being carried, the paramedic turns his seat through 180 degrees and faces backwards with the patient's head just by his knees. For the patient lying in the port bay, only his legs are inaccessible in flight. Excellent close observation and monitoring is achieved in this position (Fig. 5).

The patients are loaded through rear clam shell doors on stretchers which lock into position on runners in the cabin. In the starboard bay access to the patient is only from the mid-chest upwards and the patient lies to the side of the aircrewman out of reach of the paramedic. Seriously ill patients are not carried in this bay. Double patient carries were almost always the result of RTAs (in 21 out of 32 instances in the first 2 years) and in the event of several critically injured patients awaiting hospitalization, either some are taken by road or alternatively more than one trip is made by the Air Ambulance; the rapid journey times make this a realistic option (Fig. 6).

The use of a 'Paraid' vacuum mattress during flight makes the patient feel comfortable and is reassuring. Headphones reduce the intensity of the white noise produced by the jet engines and allow communication with the ambulance crew using a channel separate from that of the pilot. Warm blankets are provided as in winter the cabin can feel quite cold, despite the fact that the ambient temperature of the cabin maintained at about $20 \mathrm{C}$ by ducted heating. 


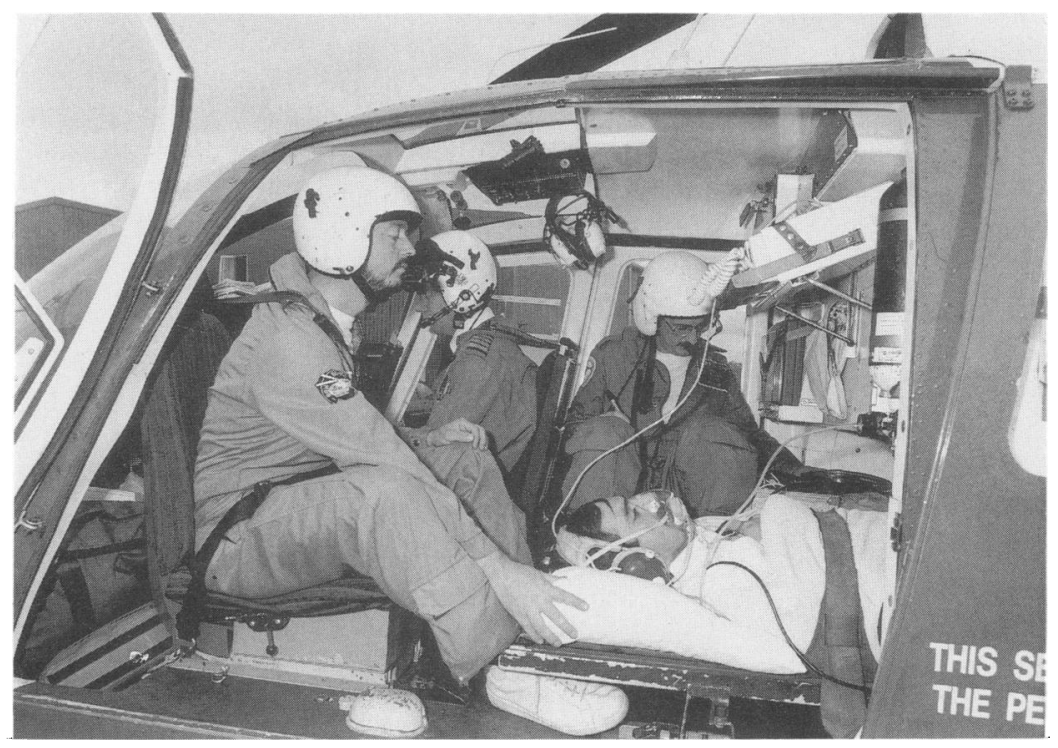

Fig. 5. Illustration showing position of patient within port bay.

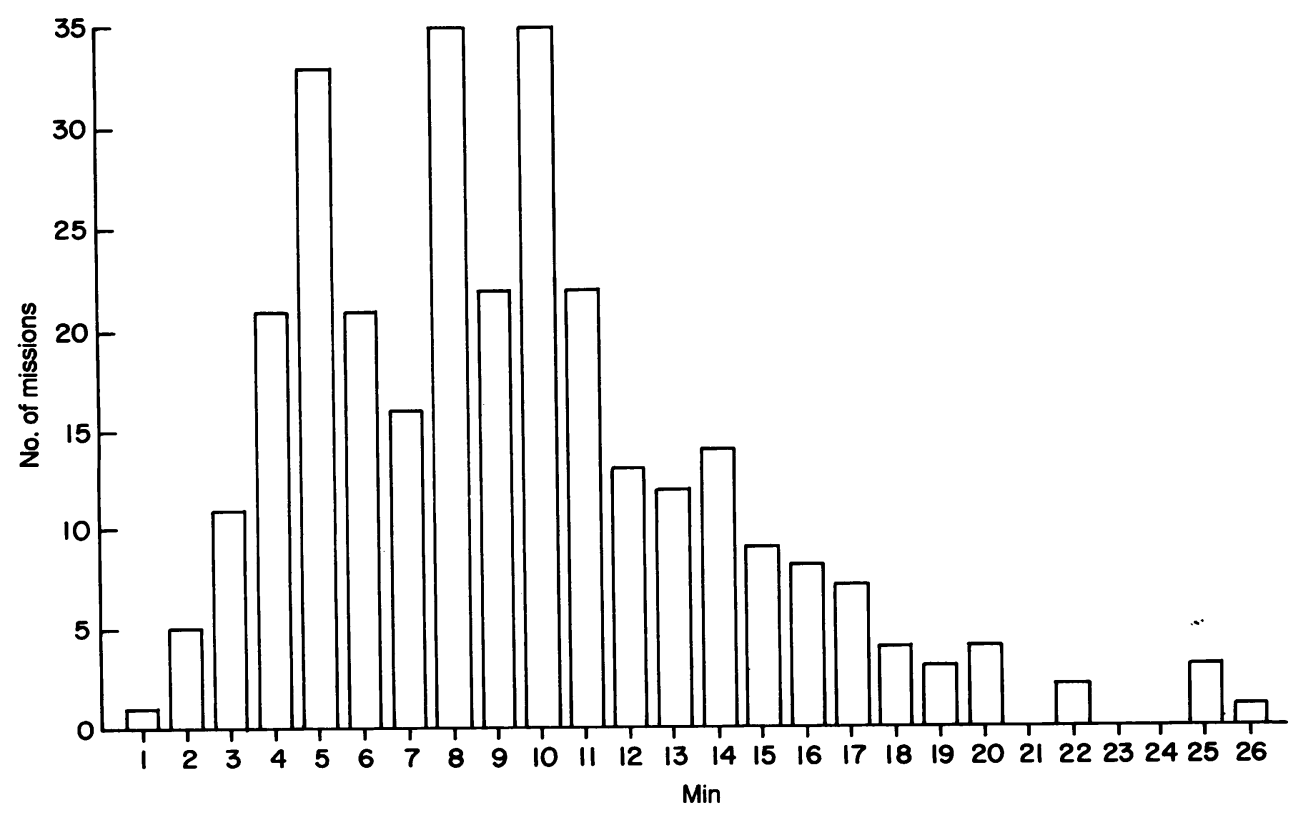

Fig. 6. Journey times of emergency trauma cases (April 1989-April 1990). 


\section{Performance}

During the hours that the Air Ambulance is available to supplement the landbased fleet, over $96 \%$ of emergency calls receive a response within $20 \mathrm{~min}$ (the ORCON standard is $95 \%$ in $20 \mathrm{~min}$ ). At other times the standard is frequently not met throughout Cornwall. (Table 1) The principal reason for failure is the wide scatter of emergency venues brought about by the simple geography and sparse population. Narrow and congested roads are an added problem. The unpredictability of emergency venues makes a solution to this problem using only land ambulances rather unrealistic: flooding the county with land ambulances would result in (1) great expense; (2) relative under-use of each resource and therefore; (3) decline in interest and standards of the crews who are under-used. The Air Ambulance offers the flexibility required in Cornwall.

The controller of the day makes an immediate decision whether to send the Air Ambulance or a road ambulance. Whichever available resource will be the first on scene is sent, usually, but other factors play a part also: (1) type of incident, if known; (2) location of incident - remote or inaccessible areas favour the helicopter whilst urban areas tend to favour a land vehicle and; (3) any need for paramedic work if not available on the land vehicle.

The activation time (time between receipt if the 999 call and the Air Ambulance being mobile) is on average $3 \mathrm{~min}$ (Fig. 7). Some of the longer times shown are produced when the Air Ambulance is not available when requested because of still being active on the last mission, refuelling etc.

In Cornwall we have emphasized the need to reduce the Therapy Free Interval (TFI) for the patient. The TFI is determined by the time lapse between the incident and the arrival of skilled help. For primary missions, when no other qualified response is made to the patient, shortening the response time (time between receipt of the 999 call and the Air Ambulance arriving on scene) will shorten the TFI. Response times for primary missions for trauma cases are shown in Fig. 8 and for responses to trauma incidents the average is under $12 \mathrm{~min}$, taking the County as a whole.

\section{On scene times}

Concern has been expressed nationally by hospital medical staff involved in trauma care about the dangers of 'overdoing' paramedical activities in the pre-

Table 1. Overall performance of the Cornwall Ambulance Service in terms of response time to emergency calls in daylight hours. Comparing 5 days of Air Ambulance availability with the 2 days when it is not available. Period: 7/3/88-15/12/88.

\begin{tabular}{ll} 
No of calls when AA available & 3509 \\
No of those calls responded to within $20 \mathrm{~min}$ & 3377 \\
$\%$ of calls responded to within $20 \mathrm{~min}$ & 96.2 \\
No of calls when AA not available & 1485 \\
No of those responded to within $20 \mathrm{~min}$ & 1401 \\
$\%$ of calls responded to within $20 \mathrm{~min}$ & 94.3 \\
\hline
\end{tabular}




\section{N. Sellwood}

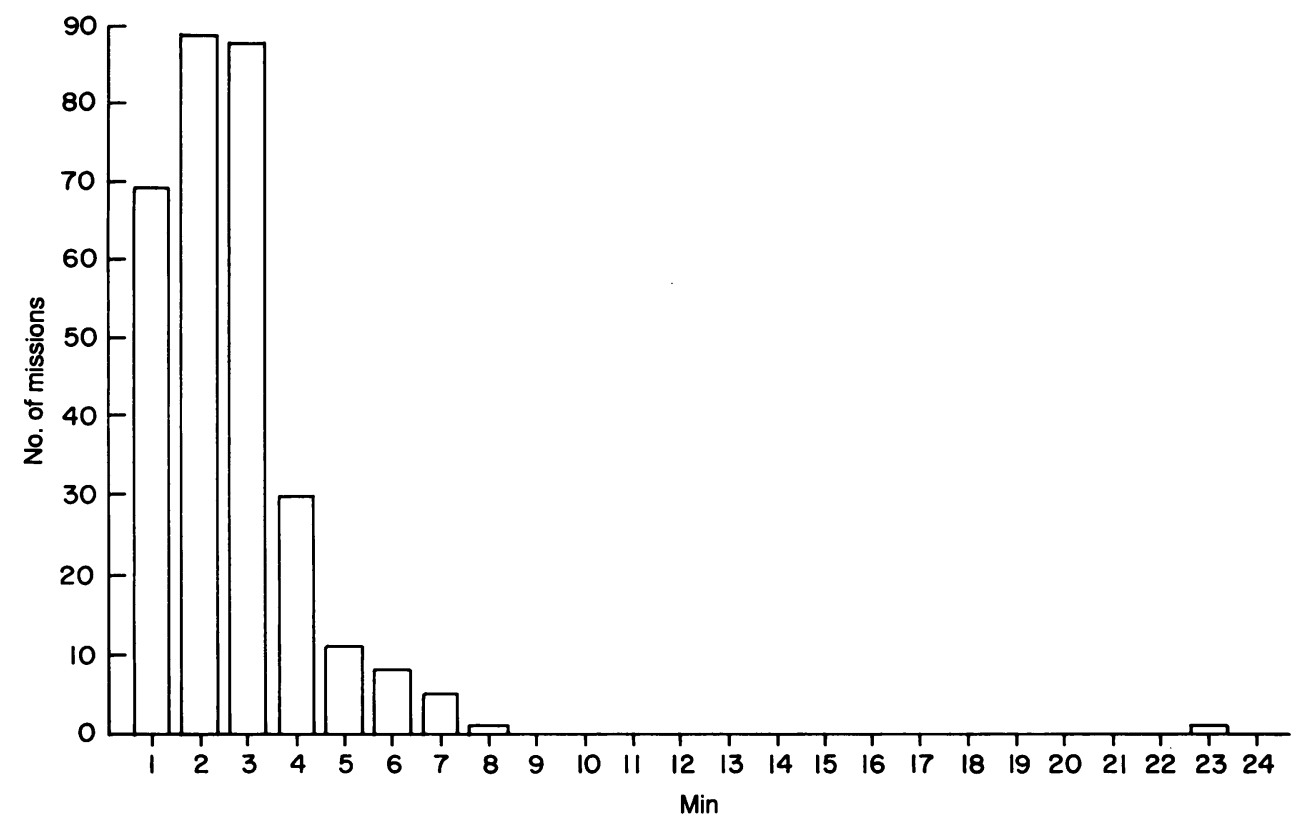

Fig. 7. Activation times (April 1989-April 1990).

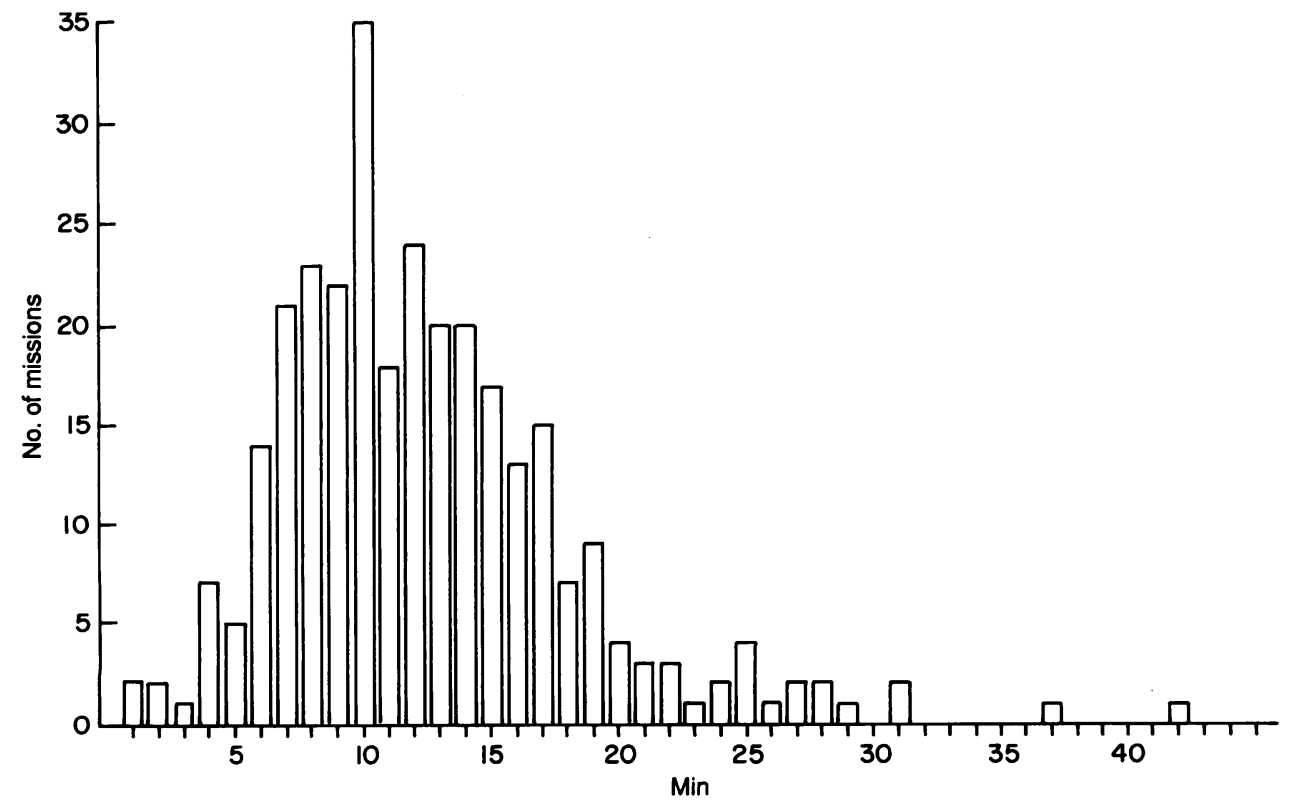

Fig. 8. Response times of emergency trauma cases (April 1989-April 1990). 
hospital phase after major trauma, causing an undesirable delay in presenting the patient at hospital for definitive treatment. Figure 9 shows the time spent by the Air Ambulance crew on scene with trauma cases and to some extent the graph demonstrates that these concerns are not unfounded. However, an average figure of $18 \mathrm{~min}$ is not wildly off the desirable, though could be improved upon.

\section{Acceptability}

In accordance with findings in other countries, patients like travelling in the Air Ambulance; there are but few exceptions (principally a fear of flying) and very few contra-indications to flying in the Air Ambulance. Flights occur at an altitude of less than 1500 feet so hypoxia due to atmospheric attenuation is not encountered, and anyway in-flight pulse oximetry, which is routine, would show up any changes in arterial oxygen saturation.

Some patients are especially suited to Air Ambulance travel because of their condition: these include head injuries, spinal injuries, unstable limb fractures, peritonitis and acute retention of urine. Comparative comfort in flight is remarked upon by patients who have experienced travel by both the Air Ambulance and land ambulance. The BO105 displays a minimum dominant resonant frequency of $26 \mathrm{~Hz}$ which, whilst appreciated as a vibration, is of low amplitude and lies outside the critical resonance frequency band of the body's organs $(6-22 \mathrm{~Hz})$ (Gillies, 1965; Gesing, 1988). This may be the reason for the relatively comfortable ride. The sensations experienced in a land ambulance of road rumble, cornering

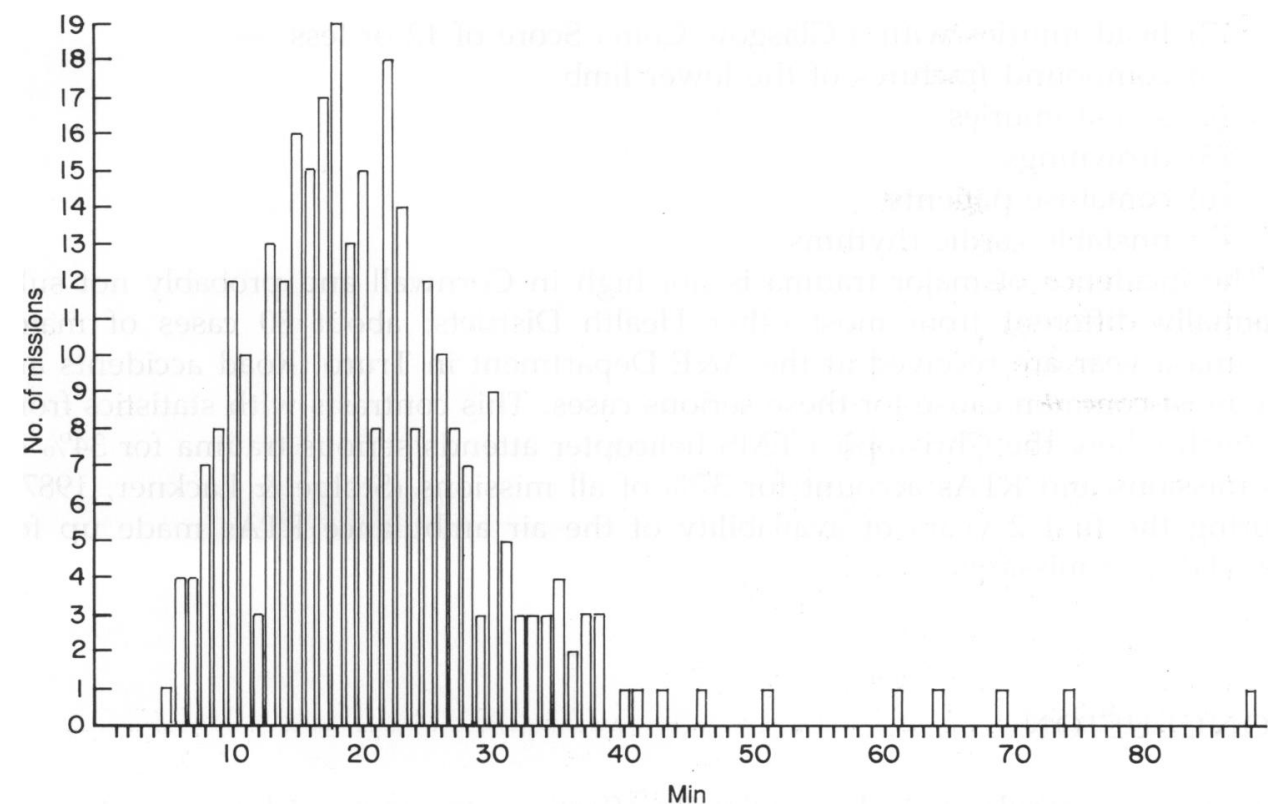

Fig. 9. Onscene times (April 1989-April 1990). 
and braking are of course absent during air flight. It must be stressed that not all helicopters exhibit favourable vibration characteristics.

Some patients whose condition does not require rapid transport are probably best taken by road. These include the very elderly, confused and restless patients and those who are mentally disturbed. Incubated babies cannot be transported without modification of the arrangements in the cabin and are currently not considered, though this does not affect the use of the Air Ambulance as a primary response vehicle.

\section{Effectiveness}

Short activation and response times have already been stressed. In addition, in $93 \%$ of cases the Air Ambulance lands within $100 \mathrm{~m}$ of the patient, so that time is not wasted in lengthy hand stretcher carries. This is especially easy in rural locations, but even in urban environments there are usually sufficient open spaces to ensure a safe landing for the Air Ambulance, such as playing fields, playgrounds, parks and even large roads.

Equally important when returning to hospital is the facility to land critically ill patients close to the A\&E department. This means that a single stretcher transfer can be effected without resorting to intermediate transport arrangements. Any variation from this can easily double or treble journey times to hospital and negates any advantages of speed which have previously been achieved. An emergency landing pad within about $100 \mathrm{~m}$ of the A\&E Department is required for appropriate care of the critically ill, but need not be used if the patient's condition is stable. Our emergency pads are used for the following categories of patients:

(1) trauma patients with a Revised Trauma Score of 10 or less

(2) head injuries with a Glasgow Coma Score of 12 or less

(3) compound fractures of the lower limb

(4) spinal injuries

(5) drownings

(6) comatose patients

(7) unstable cardic rhythms

The incidence of major trauma is not high in Cornwall and probably not substantially different from most other Health Districts; about 80 cases of major trauma a year are received at the A\&E Department in Truro. Road accidents are the most common cause for these serious cases. This contrasts with statistics from Munich where the Christoph 1 EMS helicopter attends serious trauma for $34 \%$ of its missions and RTAs account for $37 \%$ of all missions (Stolpe \& Lackner, 1987). During the first 2 years of availability of the air ambulance RTAs made up for $139(11 \%)$ of missions.

\section{CONCLUSION}

For the Air Ambulance to be maximally effective, its use must be unconstrained by adverse local arrangements such as lack of proper landing sites at hospitals. 
Although expensive to run, it can effectively cover a large geographical area which would otherwise need many land ambulances, and is therefore cost effective.

The Cornwall Air Ambulance has shown itself to be effective as a primary response emergency ambulance. It has been acceptable to patients and offers considerable advantages in critical pre-hospital care. It has shortened the Therapy Free Interval for ill patients in the Cornwall Health District and improves one of the links in the long chain of integrated care of the trauma patient.

\section{ACKNOWLEDGEMENT}

I wish to thank Paul Westaway for his help with compiling the statistics.

\section{REFERENCES}

Baxt W. G. \& Moody P. (1983) The impact of rotorcraft aeromedical emergency care services on trauma mortality. Journal of the American Medical Association 249(22), 3047-3051.

Baxt W. G., Moody B., Cleveland H. C. et al. (1985) Hospital-based rotorcraft aeromedical emergency care services and trauma mortality: a multicentre study. Annals of Emergency Medicine 14(9), 859-964.

Flora G. J. (1985) WAEDM 1, 68-70.

Gesing F. (1988) Seminar on EMS helicopters, Farnborough.

Gillies J. A. (ed) (1965) Textbook of Aviation Physiology, pp. 843.

Herve C., Gaillard M., Desfemmes C. et al. (1987) Prehospital care: the French system. Emergency Care 1, 49-53.

Hossli G. J. (1985) WAEDM 1, 70-71.

Moylan J. A. (1988) Impact of helicopters on trauma care and clinical results. Annals of Surgery 208(6), 673-678.

Stolpe E. \& Lackner C. (1987) Operating helicopter ambulances; West Germany's example. Ambulance Management International 12-15.

(Article prepared in October 1990) 